\title{
Human buildings + education
}

\author{
Y. Lopez-Wilson \\ College of Architecture, University of Nebraska, Lincoln, USA
}

\begin{abstract}
Today's intensification of social and urban change coupled with issues of sustainability amplifies the demand for architectural solutions that strengthen the balance between nature-made and man-made environments.

Wendell Berry (1990), in his book What Are People For? Calls into question the way human energies in recent history have been bent to the purposes of an extractive economy determined to dominate nature and increase the material wealth and security of our own species. Berry suggests that human welfare and meaning are located not in the values of the marketplace but in the relationships that can emerge among people and landscapes where care and continuity rather than commercial success are the central aims.

One approach to establishing "co-laboration" between the natural and built worlds is by encouraging educational experiments across the curriculum and across age groups to [explore] possible directions we might pursue in our attempt to engender a more ecologically sustainable culture. The architectural profession has undoubtedly embraced "sustainability" as the buzz word of the $21^{\text {st }}$ century. As such it has the responsibility to take a leading role in the immediate design innovations keeping in mind the economical and eco-friendliness aspects of a project without losing the archi-friendliness, as presented by University of Nebraska professor of architecture Martin Despang and graduate student Amber Ellet in their paper "Generation P(ost-fossil) fuel" to be included in the Eco Conference 2008.

This investigation takes the form of an educational experiment exploring the potential of humanoid, haptic and neural network robotics to produce a set of responsive architectural strategies to accommodate a credible range of occupancies and programs.
\end{abstract}

Keywords: eco-literacy, robots, neural networks, curriculum, typological study. 


\section{Introduction}

The first portion of this study addresses educational strategies for curriculum development in the area of architecture and robotics to promote ecological literacy in the built environment. The paper presentation will explore the great potential in developing a robotics branch to enhance the holistic approach to architectural education.

The latter is a typological study in which a specific design was developed that engenders the topics outlined in the curriculum development. The author will present by means of her own recently completed academic design project, how a young architect begins to critically evaluate the potential of robotic-enhanced structures and innovative technologies to redefine architecture within the framework of environmental design. The audience will be encouraged to consider the progressive balance of technological advances and their place in the educational framework to positively influence the delicate web of the build environment versus the natural.

\section{Curriculum introduction}

The United Nations 2002 resolution designated 2005-2014 as the Decade of Education for Sustainable Development. The initiative is supporting evidence that there is a need for universities to take the leading role and modify their curriculum to account for an ecological education. Well-respected campuses across the nation such as UC Berkeley, Rice University, UCLA, University of Virginia and the University of Texas at Austin are addressing the lack of ecological literacy in higher-level institutions by offering a degree in environmental/sustainable design to their students.

It is the belief of this author that in order to produce tomorrow's leaders in environmental stewardship, education must urge teachers and their students to thoughtfully consider aspects of our lives that either contribute or detract from the creation of an ecologically sustainable way of life.

\subsection{The need to teach robotics}

In the late twentieth century, education in its multiple forms has come to be seen as a vehicle by which individuals are able to positively influence change in their community and a place where they are provided with the tools necessary to continue to impact change in a global market.

In the past few years, important advances in computer science, artificial intelligence, biomechanics, and material science have brought to attention the world of robotics. Robots are today moving towards applications beyond the structured environment of a manufacturing plant. They are making their way into the everyday world that people inhabit. These machines are exploring distant planets, assisting with precision surgery, and locating deadly land mines [3]. 
Technology innovation is a part of human life that should be considered and Robotics is one of the fields that are currently showing the greatest advancement and development. The nature of this study calls for review, the potential of robotics in the realm of architecture. The riddle that has perplexed this author is, how can architects design buildings that offer the promise of rapid and reversible ingenious mechanical transformations in response to a changing program and occupancy pressures? The answer is believed to lie in the "crossbreeding of architecture and robotics" education.

\subsection{Analysis and definitions}

To focus the educational experience further the outlined curriculum is to center around the branches of robotics that lend themselves to an architecture that is no longer a static and passive energy consumption entity but rather an autonomous architecture that is re-generative and in constant dynamic negotiation with the energy cycles of its surroundings and with the needs of its inhabitants to seek the optimal form. It now becomes necessary to define what is meant by the terms humanoid, haptic and neural network robotics.

A humanoid robot is an autonomous robot because it can adapt to changes in its environment or itself and continue to reach its goal. This is the main difference between humanoids and other kinds of robots. In this context, some of the capacities of a humanoid robot may include self maintenance (recharge itself), autonomous learning (learn or gain new capabilities without outside assistance), adjust strategies based on the surroundings and adapt to new situations, and avoiding harmful situations to people, property and itself.

Haptic simulation is an area of robotics that focuses on the study of touch and tactile sensations, specifically as a means of communication by means of force feedback and tactile feedback. Haptic simulation experiences are common in the gaming industry. Haptic simulation is what gives the user the ability to "feel" the weight of a bowling ball or the hitting and catching of a baseball simply by holding a remote.

Neural network is a type of artificial intelligence that attempts to imitate the way a human brain works. A neural network works by creating connections between processing elements, the computer equivalent of neurons. Neural networks are particularly effective for predicting events when the networks have a large database of prior examples to draw on. Neural networks are currently used in voice recognition systems, medical imaging and aerospace applications [4].

The advances in these areas offer the greatest promise to take the built environment to the next level. With robotic technology a building could build, repair, dismantle, and recycle itself. A building equipped with sensors can track movement through a space and could adapt its shape, texture, light, sounds, and heat to the presence of a human being thereby saving exponential amounts of energy currently being used to heat, cool and maintain spaces that are not occupied by a body. 


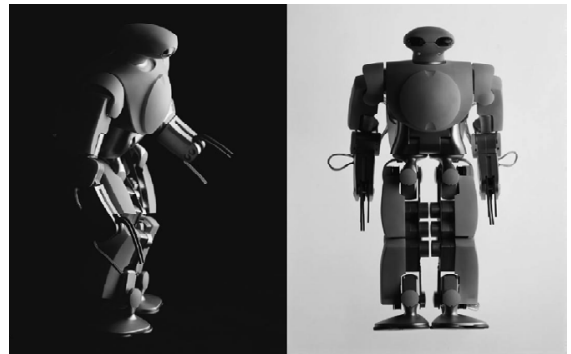

(a)

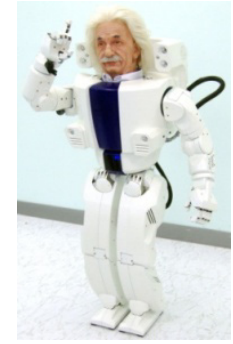

(b)

Figure 1: (a) Views of the humanoid robots morph3 in 2004 [5]. (b) Albert HUBO 2005, the latest humanoid version of the HUBO robots [6].

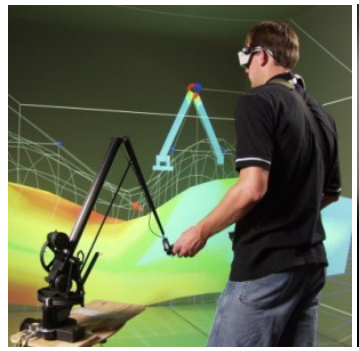

(a)

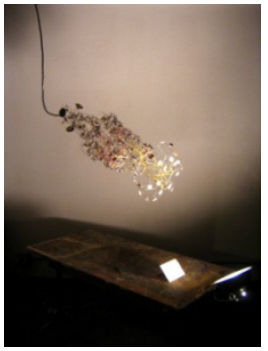

(b)

Figure 2: $\quad$ (a) Interactive Stress Reanalysis in Virtual Reality Utilizing Haptics [7]. (b) "Neural network piece" AANN Analog Artificial Neural Network [8].

Strengthening the delicate web of balance between the man-made and the nature-made can begin with the re-conceptualization of architectural education to encompass a cross disciplinary relationship between robotics and architecture to promote architectural advancement.

\subsection{Course outline}

The soul of an architect's education comes from the work developed in studio design projects. Naturally it is the suggestion of this author to begin the educational experience with a semester of studio projects with a design emphasis on the technologies aforementioned to formulate the buildings of tomorrow. The following is a partial course outline of an entire sequence of studio work meant to give the student an introductory level of knowledge: 


\section{UNIVERSITY OF NEBRASKA-LINCOLN \\ Department of Architecture}

ARCH 411

\section{Syllabus}

COURSE DESCRIPTION Architectural design focusing on understanding the fundamentals of robotics as an organizing device leading to design concepts and projects.

OBJECTIVE This course deals with questions concerning the role technological advances take on architectural ideas. The questions include the following: What is the role of technology in architecture?; How can Robotics benefit architectural design?; How can students design robots for operational simulation in their buildings?; How can students use these models to simulate a wide range of occupancies? The students will explore various areas of study in robotic technology to develop architectural designs.

METHOD Each class is composed of projects, lectures, readings, and discussions. The students are also required to participate in Kit-workshops with the Robotics and Mechatronics Lab at the University of Nebraska-Lincoln.

Kit-workshops will provide students with a more varied and open concept of robots and teach about inputs, sensing, processes, and control. The kits are comprised of structural pieces, motors, sensors, lights, interface, and programming software that will let students program and run the robot. Students will learn how to take a problem, design the robot to fulfil the need through a series of actions, program, and implement their robots.

Students may be given any real-world production problem and model its solution on the desktop. Using the kit approach, students also learn the importance of sensing and feedback and how closed loops create the accuracy of a robot. Students learn both how to program the steps and how to write programs that allow the robot to decide what to do next [9].

\section{COURSE OUTLINE}

\begin{tabular}{c} 
PART I \\
Research and Vocabulary \\
\hline
\end{tabular}

Introduction: Course Description

Vocabulary Matrix

Project 1: Research paper

Readings

Videos

\section{PART II \\ Workshops}

Kit-workshops

Guest speakers 
Project 2: Collaborate building and robot design

Discussions

Lectures

PART III
Robot Building and Final Building Design

Project 3: Independent building and robot design

Final written examination

\section{Typological study introduction}

The final part of this investigation takes the form of a typological study in which a specific design was developed that incorporates a set of kinetic responsive architectural strategies to accommodate a credible range of occupancies and programs. The concept is speculative in that it presents some of the implications of a mode of actuation that is radically different from the usual.

The idea for this design developed from the understanding of an ecosystem where all parts integrate to maintain a sometimes fragile stability. Ecosystems evolve in response to specific environmental conditions, to contain specific habitats for specific creatures and plants. If we describe buildings as an interlinked set of different habitats then we can use the idea of an ecological niche allowing us to re-examine the notion of the use of robots in the built environment, by considering them as being situated in local building habitats. For example, in nature there are areas where swamp life tribes and others where prairie life is more suited. These niches lend themselves to a specific type of use of the land to sustain a unique type of activity. These relationships are also found in buildings. A hospital can be, metaphorically speaking, like a niche where temperature, lighting, and spatial configurations are radically different than the conditions of housing units. The architect/engineer can design both robot and habitat, creating a stable ecology with a niche in which the robots might thrive to facilitate and customize the activity in these buildings to the occupants needs.

Intelligent change is the focus of the program because the idea is that buildings resemble natural ecosystems, where objects and people interact in complex rhythms according to the time of day, time of week and time of year. Furthermore, for the building to perform in a transformative and reversible manner it is evident that a level of intelligence must be present. This stresses the need for the building to have "brains" [the robots] and motor skills to communicate and engage in logical spatial functions. This argument extends to concepts of creation and decay, obsolescence and renewal. Responsive movement is the tool to address the architectural problems that static buildings can't account for and to maximize the qualities of occupant health, sustainability, versatility, durability, and longevity we seek in our built environment. For the remainder of the study the words "robots" and "brains" will be used interchangeably. 


\subsection{The "Brains" and occupant}

What developed from this notion was a specific design of a modest size building with brains: a public health educational facility. The link between a person's/scholars physical and psychological well-being and his or her academic performance is well documented, and most educators agree that a healthy environment is a key component to successful schools. However, architecture has responded in a very inappropriate way, disregarding the notion of the constant change of life, and this paper questions the competence of physical space to adapt and respond to the demands of the occupancy and improve the core vitality of public health.

Research by Bill Bordass and others has shown that many people do not like centralized management systems and want more direct control of their environment. Many management systems do not allow us to tailor the local world in any way; the management system switches the lights on, controls the blinds, the airflow, the temperature and humidity, even the smell of the air. The human component is taken out of the equation and the comfort of the environment is left to the control of the management system. Robotics offer a potential platform where the human and machine communicate succinctly to customize the users environment. It is worth mentioning that the area of central management systems has acknowledged the limitations of a "one controls all" mentality and is making strides toward independent systems.

Some buildings now include 'intelligent' components that are autonomous in their actions. A good recent example is the façade system at 'Plantation Place' designed by Arup Associates. In this system, the individual façade elements decide their local preference according to local conditions without reference to a centralized management system. This leads us to imagine the possibilities of an environment where people and individual 'active' building elements can be thought of as a wider transient population of potentially intelligent objects [10].

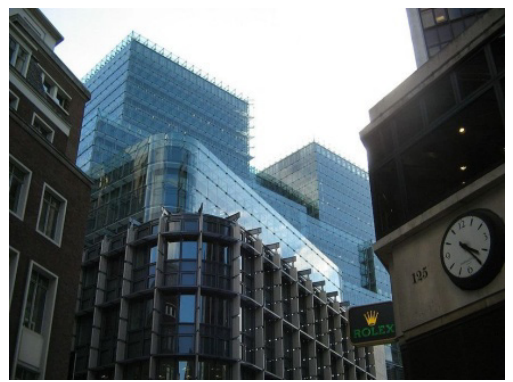

(a)

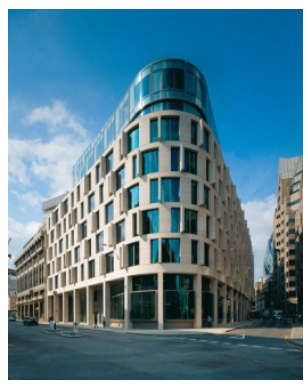

(b)

Figure 3:

(a) Plantation Place in the City of London, looking southwest from Fenchurch Street [11]. (b) Plantation Place South, London EC3 [12]. 
The College of Public Health Project proposes the use of intelligent components making use of neural network technology to facilitate the relationship between human inhabitants and robots as a symbiotic relationship of collaboration. The robots operate in a building-wide system to modify the individual spaces in response to human behavior. The robots communicate by the way that they undertake their tasks, either individually or collectively. As a result they could have positive efficiency and environmental benefits and provide entertainment and a sense of performance in twenty-first century architecture. Brains are needed to push, pull and maintain the skin of the College of Public Health Project. In this case study the student attempted to create an artificial interior landscape where sealing and floor become room partitions and seating for a lecture hall or a class room. Coincidentally if the space is no longer needed the membrane can be fully retracted to its initial state.

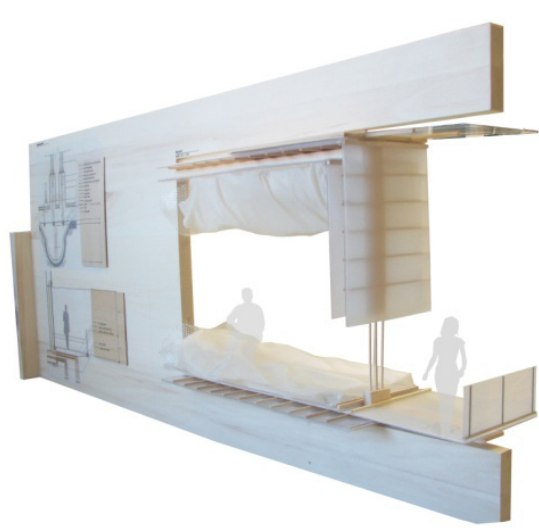

(a)

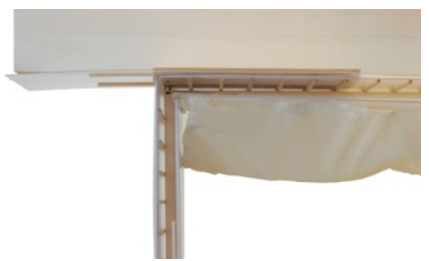

(b)

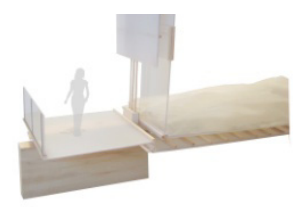

(c)

Figure 4: $\quad$ College of Public Health static model.

\subsection{Communication interface}

One of the largest conceptual steps that will be necessary to develop the idea of a brain is to trust the robot to be the local repository of the machine intelligence in the system. This means that the robot must be provided with computational ability and the necessary information to make informed decisions about its actions. This information is potentially available from a neural network computer that allows the brain to learn from previous events/stimuli. The stimuli can come from a multitude of external sources.

When nothing urgent is happening the brain will be urged to conduct an optimal 'browse' to service the boundary. The robots have individual territories defined by their personal neural network system. The brains will 'hear' all requests that are placed on them when they are in browsing mode; they ignore 
anything but immediate requests on their own territory. When overloaded they call for 'help' and initially this produces a territorial reconfiguration as help needs are evaluated collectively. Part of their function will be to gesture meaningfully to internal occupants when they are clearly wasting energy by, for example, keeping the blinds down and the lights on when this is unnecessary.

\subsection{Brain design and limitations}

The robots are equipped with moving parts (using haptic technology), a learning machine (the neural network) and sensors to behave similar to an autonomous humanoid robot. If one of these brains breaks down then there is a potential of areas left without service. Another limitation is the consumption of a certain amount of energy depending on the robots weight and the forces that they must exert during their 'work'. Their energy requirements will probably go beyond the levels of power that can be obtained through solar cells. The brains must be able to 'feed' off a power source.

\subsection{Safety and maintenance}

Graham Rowe suggests that there are inherent risks in environments that are directly occupied by people, where people pick things up and put things down in complex patterns. People are soft and extremely physically vulnerable when faced by a determined machine that has gone wrong. In this project the brains will have a job to do in a boundary zone; they will also have the power of communicating beyond it. The use of sensors in the interior lining of the membrane will send signals to the brains housed inside the membrane and outside the building to communicate the presence of a body and avoid harm.

The key to reducing risk of injury to a being or malfunction to a robot is to have parts of the brain that grip to a stable platform with power off and only release with power on. Risk is further reduced by making robots small, light and with low power actuators.

When a robot goes wrong it will malfunction neurotically by, for example, erratic movement patterns or slurred speech and it will 'freeze' and cease working. A frozen brain must be saved by a service repair robot or a human repair-man or woman who can climb in a robot environment. This means that the habitat must be to some degree 'human climbable' [10].

\subsection{Conclusion}

The concept of automation as part of the built environment has been a staple part of literature and film for over 150 years. We argue that this is the key to effective ecological architecture. Like functional human beings providing buildings with motor skills and a brain [the robots] to communicate and engage in logical spatial functions will address the architectural problems that static buildings can't account for such as occupant health, sustainability, versatility, durability, and longevity that we seek in our built environment. 
Strengthening the delicate web of balance between the man-made and the nature-made can begin with the re-conceptualization of architectural education to encompass a cross disciplinary relationship between robotics and architecture to promote architectural advancement.

The College of Public Health Project is an attempt by this author to explore the crossbreeding of architecture and robotics to design an active and responsive building as complex and intelligent as a human being. The main idea of this project focuses on a morphic interior and exterior space relationship to respond to occupancy pressures and human behavior.

\section{References}

[1] Smith, G. \& Williams, D., Ecological Education in Action: On Weaving Education, Culture, and the Environment. Albany, NY, pp. 2-6, 1999.

[2] Despang, M. \& Ellett, A. Generation P(ost-fossil). Proc. of Second International Conference on Harmonisation Between Architecture and Nature, 2008.

[3] Hayden, T., \& Hadfield, P. "The Age of Robots." U.S. News \& World Report 130, no. 16, pp.45.

[4] Oxford English Dictionary, http://0dictionary.oed.com.library.unl.edu/ entrance.dtl

[5] Okumura, Y., Tetsuo T., Takayuki F., Endo K., Masaharu S., Masaki S., \& Hiroaki Kitano. Morph3: A Compact-Size Humanoid Robot System Capable of Acrobatic Behavior. pp. 699-710, 2004.

[6] Wikipedia, http://upload.wikimedia.org/wikipedia/commons/6/66/EinsteinHubo.jpg

[7] VRAC, http://www.vrac.iastate.edu/ jmvance/RESEARCH/StressC6/ StressC6.htm

[8] Art-rash, http://www.art-rash.com/pixelform/ installation/AANN/images/ AANN-soundwalk2007-01.jpg

[9] Waddell, S., "Why Teach Robotics?" Tech Directions 58, no. 7, pp. 34.

[10] Gage, S., \& Thorne, W., "Edge Monkeys - the Design of Habitat Specific Robots in Buildings." Technoetic Arts: A Journal of Speculative Research 3, no. 3, pp. 169-179.

[11] Wikimedia, http://upload.wikimedia.org/wikipedia/commons/thumb/5/5b/ Plantation place 2.jpg/800px-Plantation place 2.jpg

[12] Britishland, http://www.britishland.com/images/PP2_01_ret2_0.jpg 